\title{
Identification of an alternative splicing signature as an independent factor in colon cancer
}

Haitao Chen ${ }^{1}$, Jun Luo ${ }^{2,3}$ and Jianchun Guo ${ }^{2,3^{*}}$ (D)

\begin{abstract}
Background: Colon cancer is a common malignant tumor with a poor prognosis. Abnormal alternative splicing (AS) events played a part in the occurrence and metastasis of the tumor. We aimed to develop a survival-associated AS signature in colon cancer.

Methods: The Percent Spliced In values of AS events were available in The Cancer Genome Atlas (TCGA) SpliceSeq database. Univariate Cox analysis was carried out to detect the prognosis-related AS events. We created a predictive model on account of the survival-associated AS events, which was further validated with a training-testing group design. Kaplan-Meier analysis was applied to assess patient survival. The area under curve (AUC) of receiver operating characteristic (ROC) was performed to evaluate the predictive values of this model. Meanwhile, the clinical relevance of the signature and its regulatory relationship with splicing factors (SFs) were also evaluated.

Results: In total, 2132 survival-related AS events were identified from colon cancer samples. We developed an elevenAS signature, in which the 5-year AUC value was 0.911. Meanwhile, the AUC values at five years were 0.782 and 0.855 in the testing and entire cohort, respectively. Multivariate Cox regression displayed that the T category and the risk score of the signature were independent risk factors of colon cancer survival. Also, we constructed an SFs-AS network based on 11 SFs and 48 AS events.

Conclusions: We identified an eleven-AS signature of colon cancer. This signature could be treated as an independent prognostic factor.
\end{abstract}

Keywords: Alternative splicing, Signature, Prognosis, Colon cancer

\section{Background}

Colon cancer is one of the most common malignancies with a high death rate [1-3]. Despite significant development in tumor screening and treatment, the overall survival (OS) rates are still low in advanced patients [4-6]. Also, the prognosis may considerably differ in colon cancer patients with similar clinical characteristics due to

\footnotetext{
* Correspondence: spring0324@126.com

${ }^{2}$ Department of Pathology, Zhongnan Hospital of Wuhan University, Wuhan 430071, China

${ }^{3}$ Wuhan University Center for Pathology and Molecular Diagnostics, Wuhan 430071, China

Full list of author information is available at the end of the article
}

the high heterogeneity [7]. Hence, unraveling the mechanism of tumor development and further uncovering novel prognostic biomarkers for prediction and therapeutic assessment is urgently required. In the past few decades, major advance has been achieved in the highthroughput technologies for colon cancer, including gene microarray, total RNA-sequence, and whole genome bisulfite sequencing [8-13]. However, these results mostly focused on the change of gene expression levels, but ignored the diversity of RNA types regulated by alternative splicing (AS).

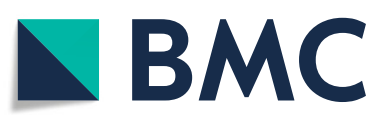

(C) The Author(s). 2020 Open Access This article is licensed under a Creative Commons Attribution 4.0 International License, which permits use, sharing, adaptation, distribution and reproduction in any medium or format, as long as you give appropriate credit to the original author(s) and the source, provide a link to the Creative Commons licence, and indicate if changes were made. The images or other third party material in this article are included in the article's Creative Commons licence, unless indicated otherwise in a credit line to the material. If material is not included in the article's Creative Commons licence and your intended use is not permitted by statutory regulation or exceeds the permitted use, you will need to obtain permission directly from the copyright holder. To view a copy of this licence, visit http://creativecommons.org/licenses/by/4.0/ The Creative Commons Public Domain Dedication waiver (http://creativecommons.org/publicdomain/zero/1.0/) applies to the data made available in this article, unless otherwise stated in a credit line to the data. 
The process of AS creates considerable biological complexity from a limited number of genes, and its disorder often leads to disease [14]. The AS changes observed in tumors may represent an independent carcinogenic process and may be related to the functional transformation of cancer [15]. Also, accumulating evidence has discovered that the aberrant AS events were highly associated with the occurrence and metastasis of some cancers [16-19]. Previous articles [20-23] had identified some AS events for the prognosis assessment of colorectal cancer. However, the contribution of AS to colon cancer is not fully understood. Also, the prognostic model in these papers lacks validation. Recently, Zhang et al. [24] also built an AS signature to predict the relapse of I-III colon cancer.

The present study aimed to identify and validate an AS signature for colon cancer based on the survivalassociated AS events. The predictive values of the model were further evaluated. Additionally, the clinical relevance of this model and its regulatory relationship with splicing factors (SFs) was also assessed.

\section{Methods}

\section{Data acquisition}

We obtained the transcriptome data and survival data of colon cancer from The Cancer Genome Atlas (TCGA) database. SFs list and the Percent Spliced In (PSI) values for AS events were collected from the SpliceAid 2 database [25] and TCGA SpliceSeq [26], respectively. To obtain reliable information of ASs, only samples with a PSI value $>75 \%$ were included for further analysis [24]. Seven different subtypes of AS events were discovered, involving alternate acceptor site (AA), alternate donor site $(\mathrm{AD})$, alternate promoter $(\mathrm{AP})$, alternate terminator (AT), exon skip (ES), mutually exclusive exons (ME), and retained intron (RI).

\section{Prognosis-associated AS events}

To exclude the influence of short-term follow-up on prognosis of colon cancer, samples without follow-up information or with follow-up less than 90 days were excluded. The function of impute.knn() with the impute package using R software (3.6.1) was used to replenish the missing data. When PSI value $<0.05$ or the standard deviation of PSI value in all samples is less than 0.01 , the AS data were also deleted. We carried out univariate Cox regression analysis to detect the survival-associated AS events, which were presented with the UpSet map and the volcano plot. Similarly, the first 15 AS events from the seven subtypes were displayed in the bubble chart.

\section{Identification of a prognostic AS signature}

After data filtering, we randomly divided the remaining colon cancer samples into the training and testing cohorts. We conducted Lasso regression analysis to avoid the overfitting of the signature in the training cohort. Furthermore, multivariate Cox regression analysis was carried out to detect the ultimate prognostic AS events of the signature. The risk score was acquired according to the following formula:

$$
\text { Risk score }=\sum_{\mathrm{i}=1}^{\mathrm{N}}(\mathrm{PSI} * \text { Coei })
$$

In the training cohort, we randomly divided colon cancer patients into the high-risk and low-risk subgroups based on the median of the risk score. We performed Kaplan-Meier analysis to compare the OS between the high-risk and low-risk subgroups. Moreover, the time receiver operating characteristic (ROC) analysis was conducted to evaluate the prognostic signature. An area under the curve (AUC) $>0.75$ was considered suitable for predictions. Also, the risk score distribution map, the survival status map, as well as the heatmap of PSI values were used to assess this AS signature.

\section{Validation of the signature}

We applied the testing and entire cohorts to validate the reliability of the signature. Furthermore, we conducted survival analysis and ROC analysis to assess the signature. The risk score analysis of AS events was also applied to evaluate this signature. $P<0.05$ for survival analysis and AUC $>0.6$ was accepted for predictions.

\section{Applicability of the signature}

To measure the prognostic value of the AS signature, we analyzed the clinical prognostic factors, including age, gender, the pathological stage, the $\mathrm{T}$ category (assessing the invasion of the tumor), the $\mathrm{M}$ category (assessing the distant metastasis of the tumor), the $\mathrm{N}$ category (assessing the lymph node metastasis of the tumor), and the risk score of the signature. In univariate and multivariate analysis, when $p<0.05$, these factors were considered as independent prognostic variables. The relationships between the signature and the clinical features in the entire cohort were also evaluated.

\section{Construction of an SFs-AS network}

We applied Spearman test to evaluate the correlation between the survival-associated AS events and SF genes. Correlation coefficient $>0.5$ and $p<0.001$ was the cutoff values. Subsequently, we developed an SFs-AS network, including the prognosis-associated AS events and related SFs. Moreover, Cytohubba plug-in was applied to identify the hub nodes based on eleven algorithms (seven global-based and four local-based methods). 


\section{Results}

\section{Data acquisition}

In total, 452 samples with 473 expression profiles were involved in the present study. We collected AS event profiles of 443 colon cancer samples from the TCGA SpliceSeq data portal [26]. 35,391 AS events from 17,401 genes were identified, including $7740 \mathrm{AT}$ in 3381 genes, 2917 AA in 2124 genes, $2524 \mathrm{AD}$ in 1833 genes, 6653 AP in 2692 genes, 13,087 ES in 5634 genes, $138 \mathrm{ME}$ in 137 genes, and 2332 RI in 1600 genes (Fig. 1a). Overlaps of the seven subtypes of AS events were depicted in the UpSet plot (Fig. 1c). This indicates that one gene could own multiple kinds of mRNA splicing events. Among all these AS events of colon cancer, ME was the least common type, while ES was the most.

\section{Prognosis-associated AS events}

The survival-associated AS events of colon cancer were discovered by univariate Cox regression analysis. One sample lacking of follow-up data and 56 samples with follow-up less than 90 days were ruled out. Fifteen samples with a small standard deviation of the PSI values were also deleted. The prognosis-associated AS events from the remaining 380 patients were studied (Table S1). In total, 2132 AS events with 1647 genes were remarkably associated with OS $(p<0.05)$. Thus, one gene
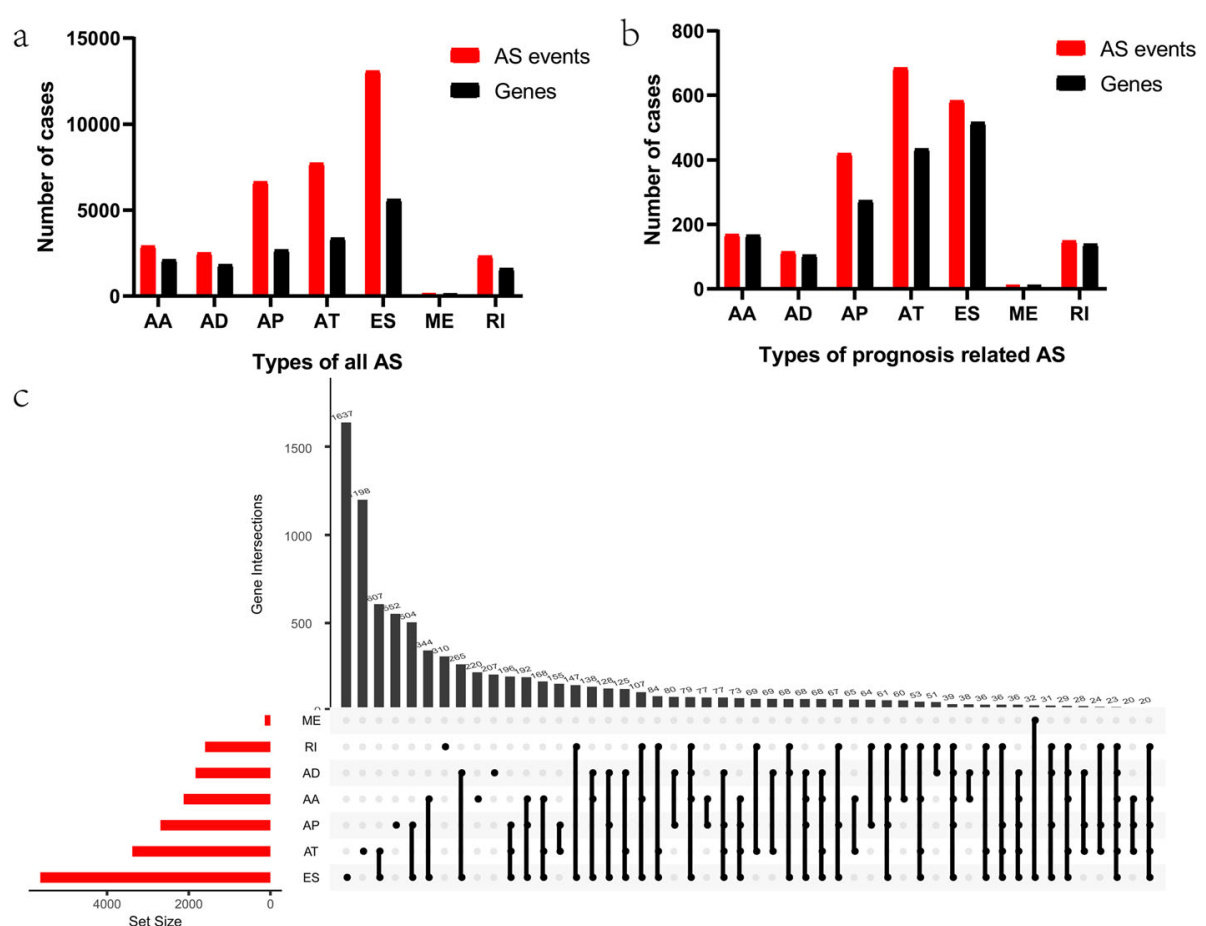

ypes of prognosis related AS
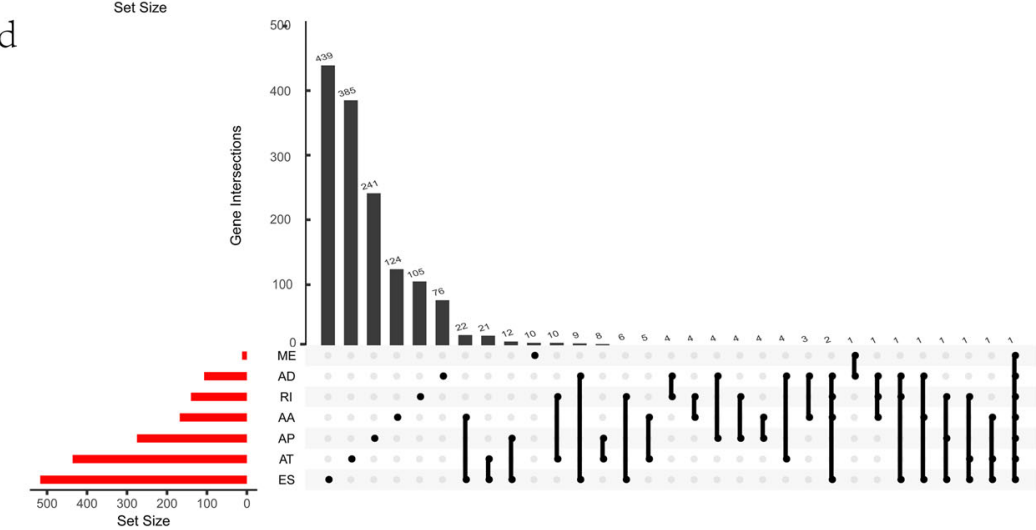

Fig. 1 Alternative splicing (AS) events of colon cancer. a Numbers of all AS events and genes in 443 colon cancer patients. b Numbers of prognosis-related AS events and genes in 380 colon cancer patients. c Upset plot of all AS events. d Upset plot of survival-related AS events. AA, alternate acceptor site; AD, alternate donor site; AP, alternate promoter; $\mathrm{AT}$, alternate terminator; $\mathrm{ES}$, exon skip; $\mathrm{ME}$, mutually exclusive exons; RI, retained intron 


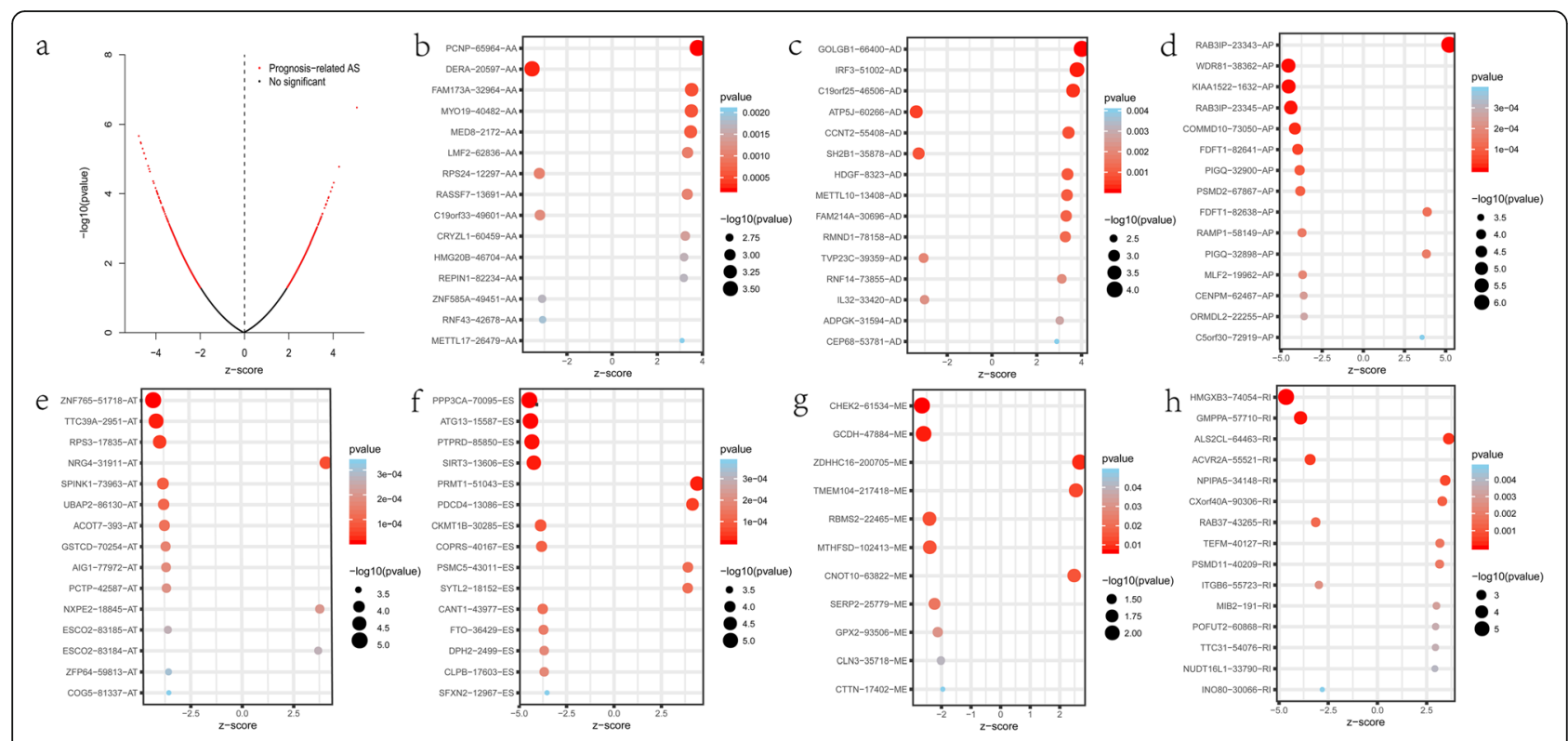

Fig. 2 Survival-associated alternative splicing (AS) events in the colon cancer cohort. a The distributions of survival-related AS events in volcano plot. $\mathbf{b}$-h Forest plots of the top 15 survival-related AS events for seven splicing subtypes

might have several AS events, among which ES was the predominant ones (Fig. 1b, d). The AS events were displayed in the volcano map (Fig. 2a). The first 15 survival-associated AS events from the seven types were exhibited in Fig. 2b-h.

\section{Identification of a prognostic AS signature}

We applied a training-testing group scheme and the 380 colon cancer samples were randomly separated into the training and testing groups (Table S2). We get eighteencandidate prognostic AS events by conducting Lasso regression in the training group (Fig. 3a, b). Next, we carried out multivariate Cox analysis to acquire eleven optimal survival-related AS events, including WDR81-38362-AP, KIAA1522-1632-AP, PPP3CA-70,095-ES, ATG13-15587ES, SIRT3-13606-ES, COMMD10-73050-AP, PDCD413086-ES, NRG4-31911-AT, GMPPA-57710-RI, CKMT1B30,285-ES, and PIGQ-32900-AP. Among these AS events, PDCD4-13086-ES and NRG4-31911-AT are high hazard, while the remaining AS events being low hazard. The details of these prognostic AS events in the model of colon cancer were presented in Table 1. Based on the median value of the
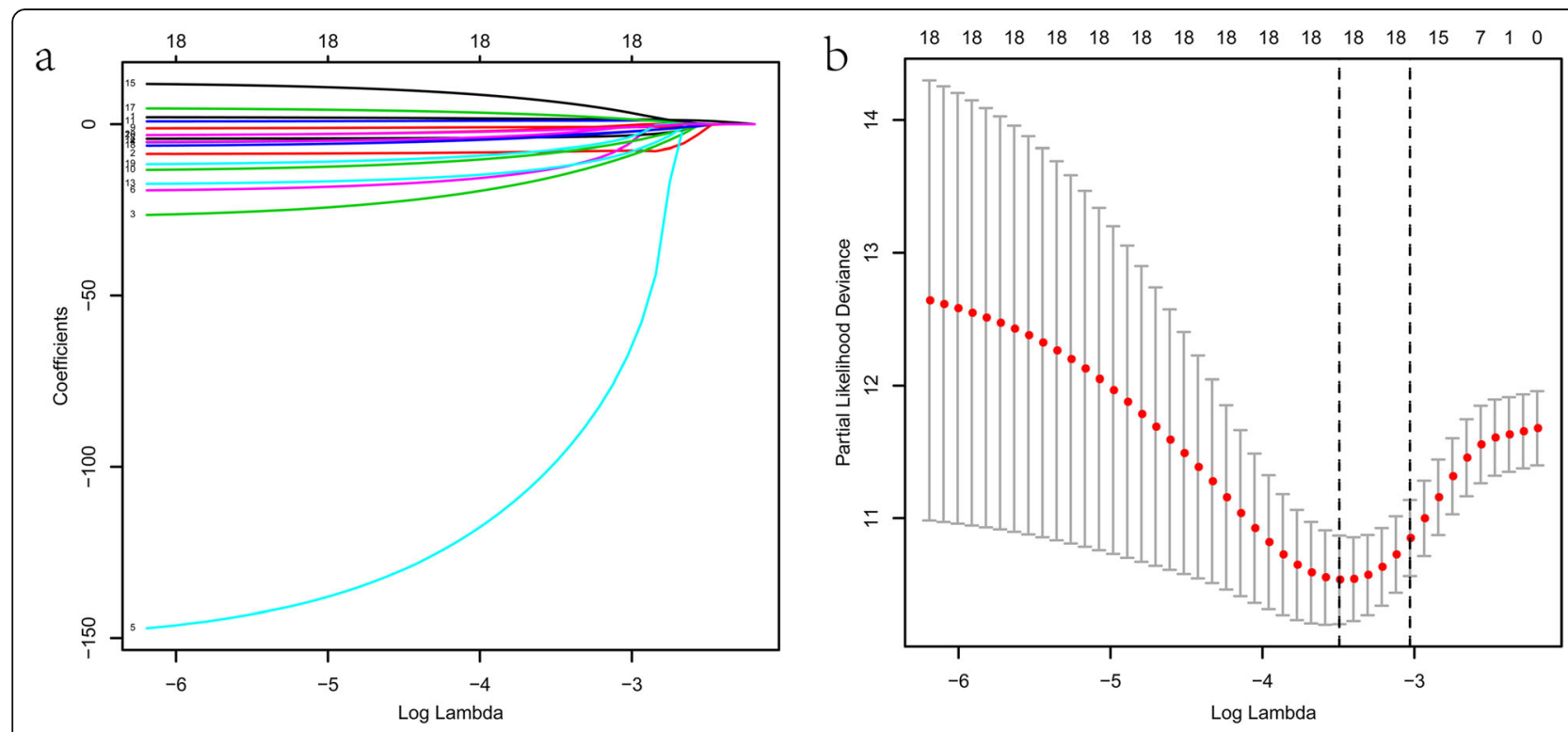

Fig. 3 Lasso regression analysis. a LASSO coefficient. b A graph of the error rate of cross-validation 
Table 1 Prognostic index for colon cancer patients based on survival-related AS events

\begin{tabular}{llllll}
\hline AS events & Coef & HR & HR.95 L & HR.95H & $\boldsymbol{p} v a l u e$ \\
\hline WDR81-38362-AP & -27.6419 & $9.89 \mathrm{E}-13$ & $1.15 \mathrm{E}-16$ & $8.48 \mathrm{E}-09$ & $2.20 \mathrm{E}-09$ \\
KIAA1522-1632-AP & -6.41637 & 0.001635 & 0.000139 & 0.019261 & $3.43 \mathrm{E}-07$ \\
PPP3CA-70,095-ES & -151.93 & $1.04 \mathrm{E}-66$ & $1.74 \mathrm{E}-88$ & $6.24 \mathrm{E}-45$ & $2.88 \mathrm{E}-09$ \\
ATG13-15587-ES & -21.3707 & $5.23 \mathrm{E}-10$ & $1.28 \mathrm{E}-13$ & $2.13 \mathrm{E}-06$ & $4.70 \mathrm{E}-07$ \\
SIRT3-13606-ES & -19.6613 & $2.89 \mathrm{E}-09$ & $1.38 \mathrm{E}-12$ & $6.06 \mathrm{E}-06$ & $4.68 \mathrm{E}-07$ \\
COMMD10-73050-AP & -22.1589 & $2.38 \mathrm{E}-10$ & $9.79 \mathrm{E}-15$ & $5.78 \mathrm{E}-06$ & $1.70 \mathrm{E}-05$ \\
PDCD4-13086-ES & 16.55192 & $15,431,416$ & $39,384.6$ & $6.05 \mathrm{E}+09$ & $5.53 \mathrm{C}-08$ \\
NRG4-31911-AT & 4.79629 & 121.0605 & 18.34399 & 798.9342 & $6.30 \mathrm{E}-07$ \\
GMPPA-57710-RI & -6.01656 & 0.002438 & 0.000161 & 0.03691 & $1.43 \mathrm{E}-05$ \\
CKMT1B-30,285-ES & -13.7515 & $1.07 \mathrm{E}-06$ & $4.16 \mathrm{E}-09$ & 0.000273 & $1.17 \mathrm{E}-06$ \\
PIGQ-32900-AP & -3.86593 & 0.020944 & 0.002297 & 0.190962 & 0.000608 \\
\hline
\end{tabular}

AS alternative splicing

risk score, colon cancer patients were subsequently classified into the high-risk and low-risk subgroups. There was significant difference in the ROC analysis between the two groups $(p=6.721 \mathrm{e}-12)$ (Fig. 4a). The AUC values of OS for the eleven-AS events prognostic model at 3- and 5-year was 0.895 (95\% confidence interval: 0.809-0.981) and 0.911 (95\% confidence interval: 0.819-1), respectively (Fig. 4b). Risk score distribution map, the survival status map, and the heatmap of PSI values were shown in Fig. 4c-e.

\section{Validation of the signature}

To confirm the usability of this signature, we validated it using the validating groups. These two validating cohorts were randomly separated into two groups on the basis of

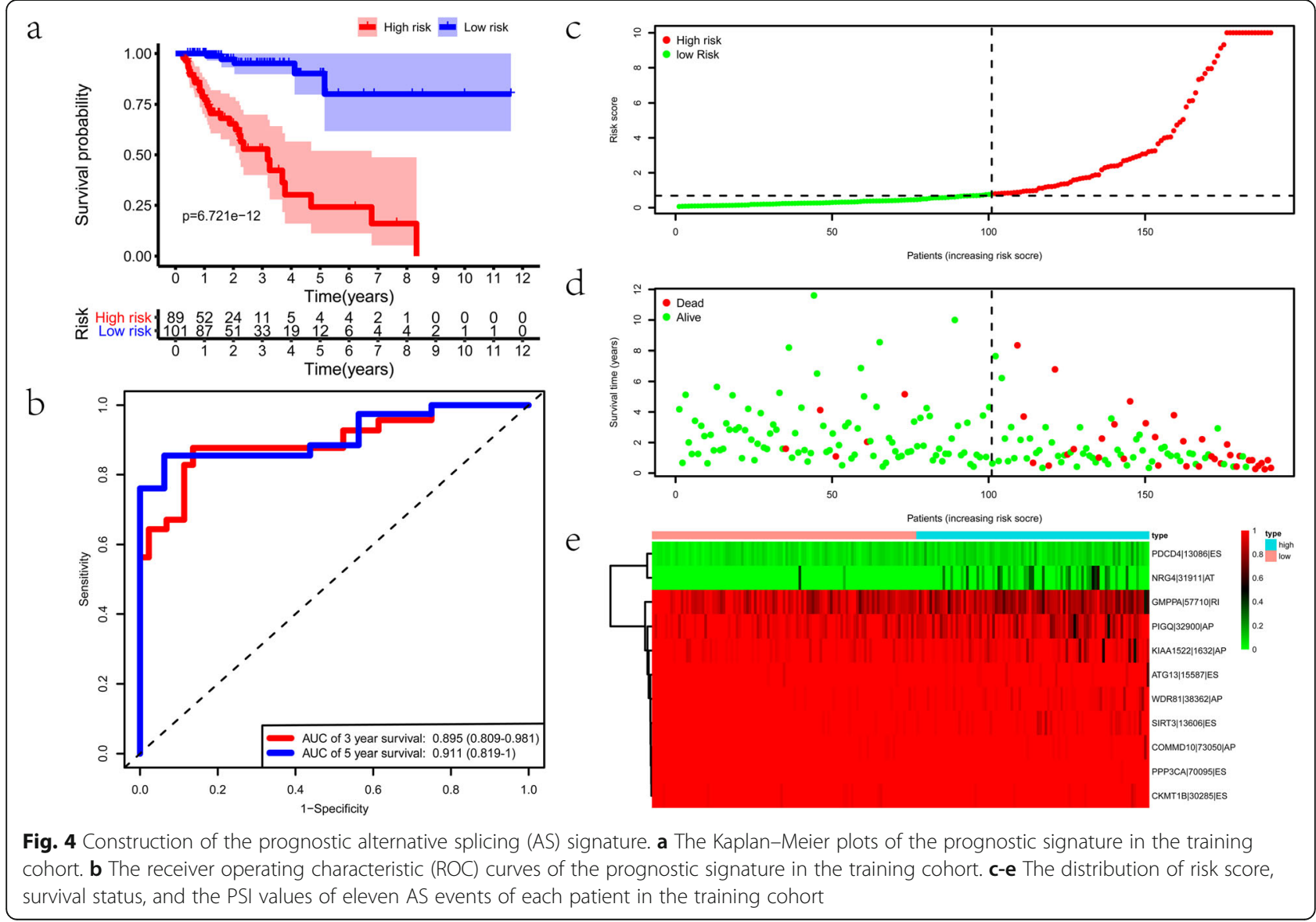


the risk score. We found significant difference in OS between the two risk groups in both cohorts (all $p<0.05$ ) (Fig. 5a, b). The AUC values of both cohorts $>0.75$ (Fig. $5 c, d$ ), which indicates that this signature could accurately predict the prognosis of colon cancer. Likewise, the risk curve of AS events was presented in Fig. 6a-f. All these results revealed that the AS signature was reliable in predicting the prognosis of colon cancer.

\section{Applicability of the signature}

Several clinical parameters, including the pathological stage, the $\mathrm{T}$ category, the $\mathrm{M}$ category, the $\mathrm{N}$ category, and the risk score were identified, which could predict the survival of colon cancer patients (Fig. 7a). The $\mathrm{T}$ category and the risk score of this signature were independent risk factors according to multivariate analysis (Fig. 7b).
Then, we found that the risk score of the signature was better than the $\mathrm{T}$ category in predicting the fiveyear OS (Fig. 7c). Next, we estimate the correlation of the signature with other clinical variables (Table S3). GMPPA-57710-RI was a low hazard AS event, while NRG4-31911-AT and PDCD4-13086-ES were highhazard AS events. The PSI value of GMPPA-57710-RI was considerably lower in patients with higher pathological stage, higher $\mathrm{M}$ category, higher $\mathrm{N}$ category, and higher $\mathrm{T}$ category (Fig. 8a-d). On the contrary, the PSI value of NRG4-31911-AT was substantially higher in patients with a higher pathological stage. (Fig. 8e). The PSI value of PDCD4-13086-ES was markedly higher in patients with higher pathological stage, higher $\mathrm{M}$ category, and higher $\mathrm{N}$ category (Fig. 8f-h). Additionally, the risk score was higher in patients with a higher $\mathrm{T}$ category than those with a lower T category (Fig. 8i). These

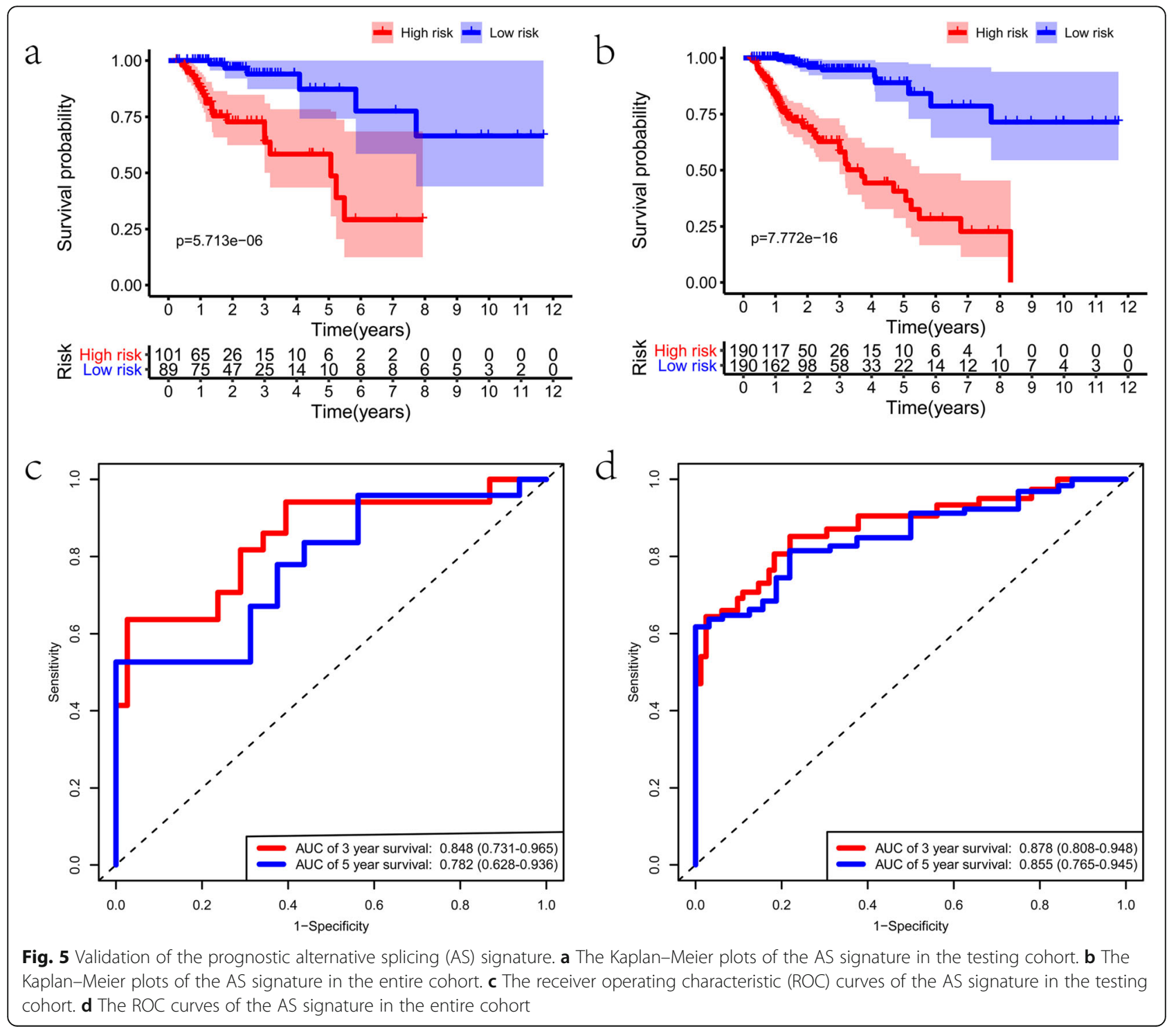



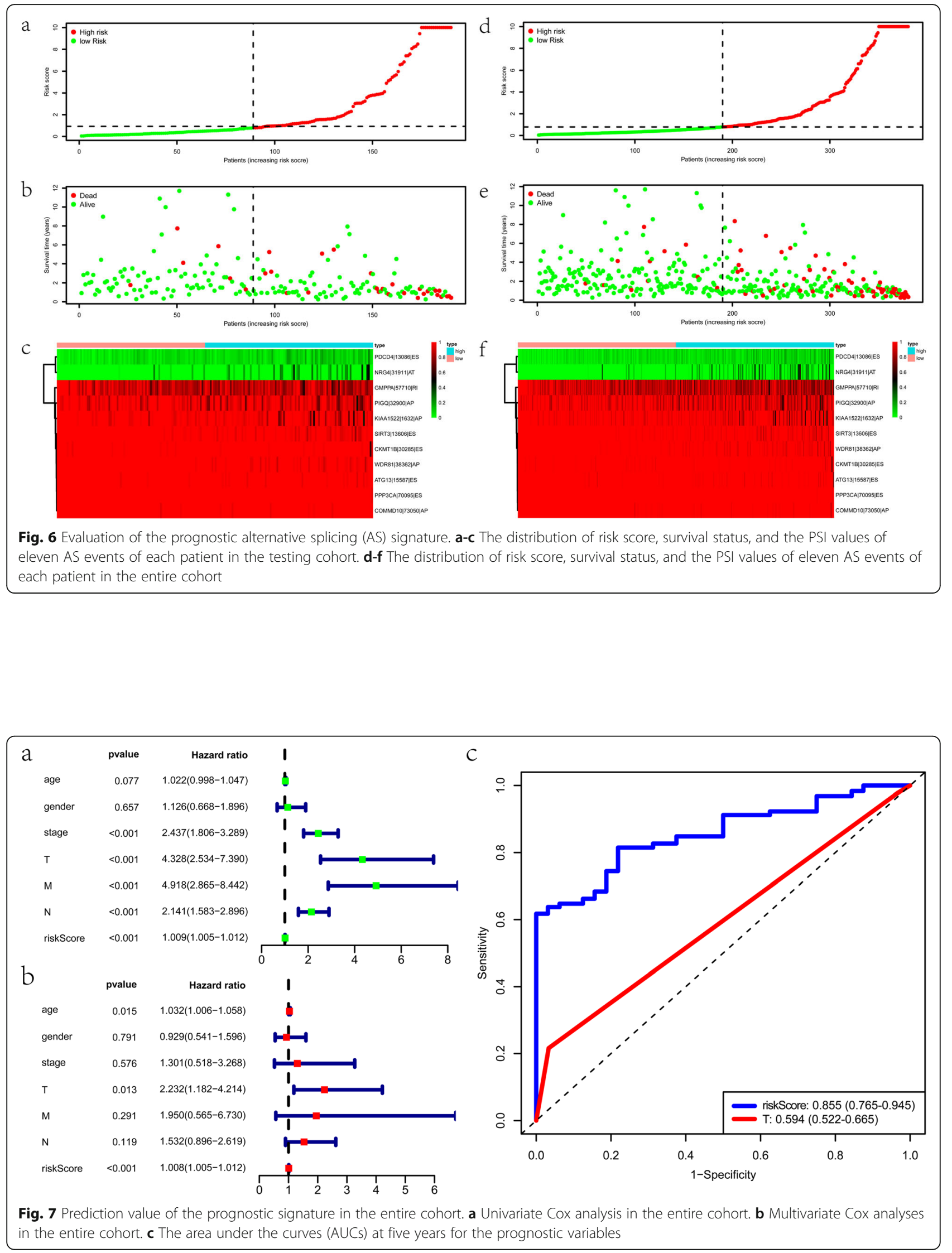


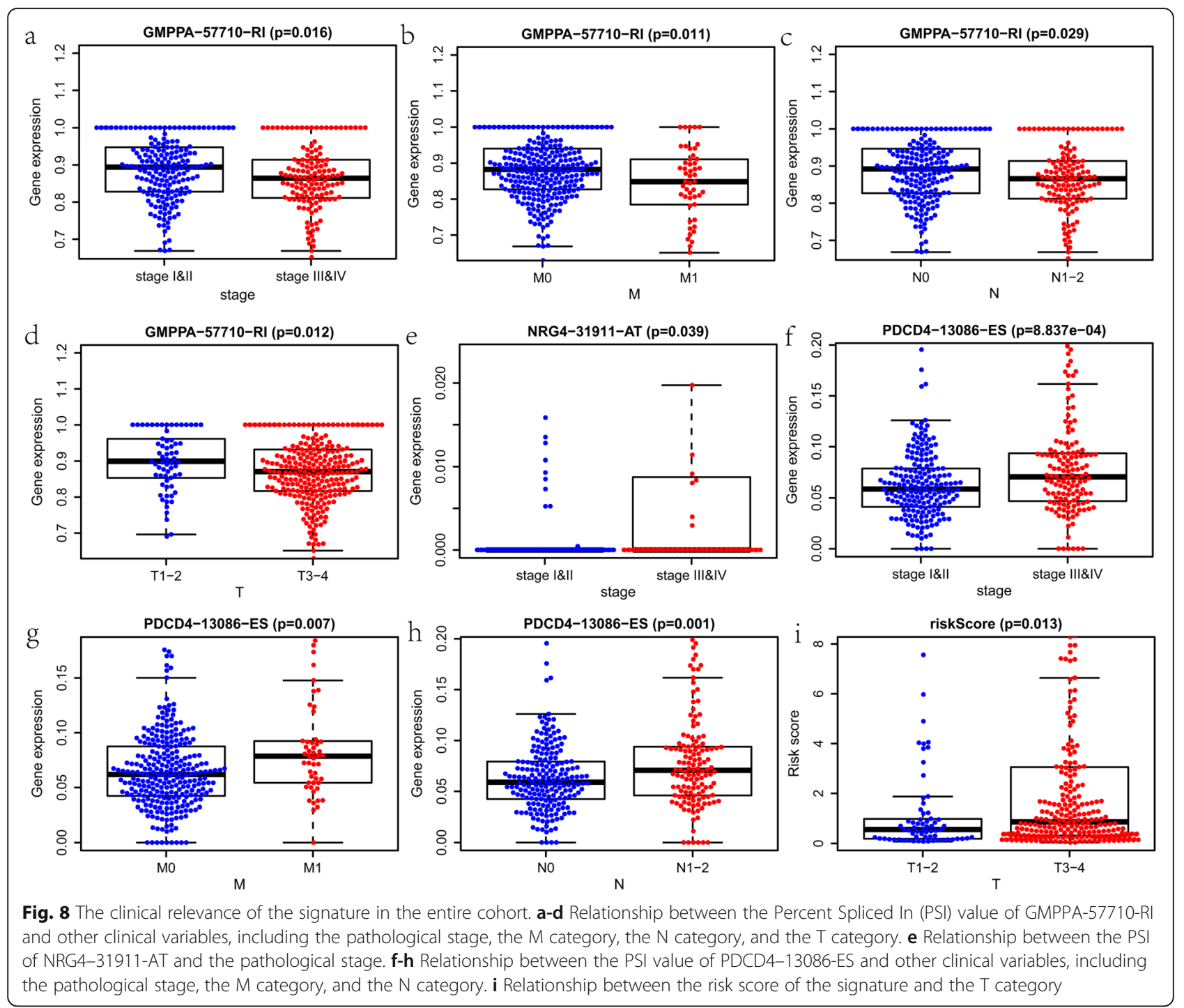

results above demonstrated that the model is a reliable and independent prognostic factor of colon cancer.

\section{Development of an SFs-AS network}

We firstly identified 17 survival-associated SFs. The survival-associated AS events and the survival-related SFs were detected when correlation coefficient $>0.5$ and $p<0.001$, which represented a moderate correlation. Then, we rebuilt an SFs-AS network based on these survival-associated ASs and SFs, including 30 adverse AS events (red triangles), 27 favorable AS events (green triangles), and 6 SFs (blue circulars) (Fig. 9a). Several SFs, including CLK2, CWC22, INTS3, and XAB2 were linked with worse survival of patients, while LSM2 and PPP1CA were associated with favorable prognosis (Fig. 9b-d and Fig. S1). Also, we found that most favorable AS events were positively correlated with SFs of good survival, while most adverse AS events were positively associated with SFs of poor survival. Furthermore, based on the network, we detected five hub nodes, including one adverse AS event (RPAIN-38678-AT), one favorable AS event (MRPL20-165-AT), and three SFs (CLK2, INTS3, and XAB2). Correlation analysis showed that the expression of CLK2 and INTS3 were positively associated with PSI values of RPAIN-38678-AT, while the expression of INTS3 were negatively linked with PSI values of MRPL20-165-AT (Fig. 9e-g).

\section{Discussion}

Colon cancer is a common malignant tumor with a poor prognosis. Abnormal AS events were reported to play crucial roles in the development of several cancers [2729], which might be treated as a potential biomarker. In the present study, we detected 2132 prognosisassociated AS events from the TCGA SpliceSeq database. Then, we identified an eleven-AS signature and 

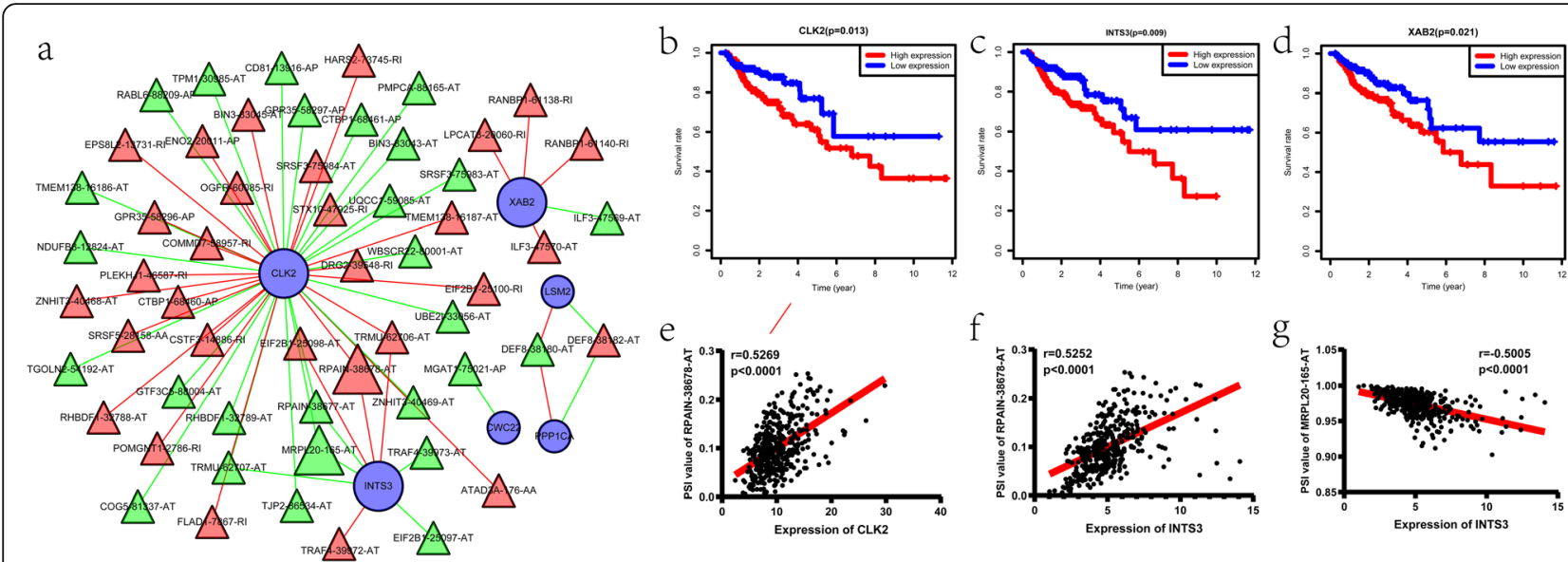

Fig. 9 Construction of a splicing factors (SFs)-alternative splicing (AS) network. a The SFs-AS network. b-d Kaplan-Meier curves for CLK2, INTS3, and XAB2 with high (red) and low (blue) expression group in colon cancer. e Correlation between expression of CLK2 and PSI value of RPAIN38678-AT. $\mathbf{f}$ Correlation between expression of INTS3 and PSI value of RPAIN-38678-AT. $\mathbf{g}$ Correlation between expression of INTS3 and PSI value of MRPL20-165-AT. For a, blue circulars represent the survival-related SFs; red triangles and green triangles represent diverse AS events and favorable AS events, respectively; red lines and green lines represent the positive and negative relationship between Percent Spliced In (PSI) values of AS events and SFs

used the validated cohorts to evaluate the model. Next, we developed an SFs-AS network with the survivalrelated AS events and related SFs. Furthermore, weevaluated the prediction efficiency of the signature, which could be treated as an independent prognostic factor.

Devaud et al. [30] revealed that several AS variants of FAK might be used as potential biomarkers and treatment targets in the development and metastasis of colorectal cancer. Flodrops et al. [31] found that TIMP1 intron 3 retention could affect the progression of colon cancer. Huang et al. [32] constructed an AS signature based on differentially expressed AS events between leftand right-sided colon cancer. In the present study, we detected eleven AS events for the construction of the model. It was reported that SIRT3 silencing could be a therapeutic strategy to render colon cancer cells more sensitive to treatment [33]. In this study, SIRT3-13606ES was a favorable AS event. This means that the ES of SIRT3 functions as a tumor suppressor in colon cancer. PDCD4 could overcome the resistance to an IGF-1R/IR Inhibitor in colon carcinoma cells, which could be used for the treatment of colon cancer [34]. In this study, PDCD4-13086-ES is a diverse AS event, which means that the ES of PDCD4 plays roles as an oncoprotein in colon cancer. The role of the remaining protein in the model of colon cancer remains not clear. However, these multiple prognosis-related AS events may partially elucidate the heterogeneity of colon cancer and contributed to the treatment of colon cancer.

Previous studies have created some molecular signatures of colon cancer. Lv et al. [35] constructed a fivelncRNA signature to predict the OS of colon cancer patients. We previously identified a five-immune gene signature for colon cancer, which contributed to its early diagnosis and prognostic prediction [36]. Similarly, Wang et al. [37] identified an epigenetic methylationdriven signature, which was associated with survival for colon cancer. In this study, we identified an eleven-AS signature of colon cancer according to the prognosisrelated AS events. The AUCs of OS for this model at 5year were larger than 0.9 , which demonstrated its excellent prediction value. Additionally, the AUCs of the prognostic AS model at 5-year were larger than 0.75 in the validating cohorts, which proved the reproducibility of this model. Furthermore, the superiority of the model to other clinical parameters made this signature a better independent prognostic factor. Also, the consistence of risk score of this model with other clinical outcomes further verify the reliability of this signature.

AS changes may originate from expression changes in SFs, which affect the splicing of cancer-related genes $[38,39]$. SFs could affect the specific binding of spliceosome to pre-mRNA sequences, thus generating vast and diverse mature mRNAs [14]. Furthermore, SFs function as oncoproteins or tumor suppressors and could be used as a drug target in cancer therapy $[40,41]$. Chen et al. [42] demonstrated that the disordered expression levels of SFs might influence the pathogenesis of cancer. Thus, it is crucial to discover the regulatory network between the prognosis-associated AS events and the related SFs. In the present study, we constructed an SFs-AS regulatory network based on the prognosis-related AS events and SFs. We identified three hub SFs, including CLK2, INTS3, and XAB2. Until now, the role of these SFs in colon cancer is unclear. Based on our network, CLK2, 
INTS3, and XAB2 may function as oncoproteins because that the high expression of these SFs showed poor survival of colon cancer. In addition, these genes were positively corelated with diverse AS events. However, their potential roles in the occurrence and progress of colon cancer remains to be studied.

This study has several limitations. First, the prognostic AS signature was developed according to public databases, which needed further verification by future clinical researches. Second, the relationship between the survival-related AS events and their corresponding SFs, as well as the underlying mechanisms behind the development of colon cancer, requires further study.

\section{Conclusion}

We identified an eleven-AS event signature. This signature could be treated as an independent prognostic factor.

\section{Supplementary information}

Supplementary information accompanies this paper at https://doi.org/10. 1186/s12885-020-07419-7.

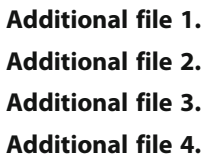

\section{Abbreviations}

OS: Overall survival; AS: Alternative splicing; SFs: Splicing factors; TCGA: The Cancer Genome Atlas; PSI: Percent Spliced In; AA: Alternate acceptor site; AD: Alternate donor site; AP: Alternate promoter; AT: Alternate terminator; ES: Exon skip; ME: Mutually exclusive exons; RI: Retained intron; ROC: Receiver operating characteristic; AUC: Area under the curve

\section{Acknowledgments}

None.

\section{Authors' contributions}

HTC and JCG conceived the study and performed the bioinformatics analyses. HTC and JL downloaded and organized the clinical and gene expression data. HTC and JCG performed the statistical analyses. HTC and JCG wrote the manuscript. JL and JCG critically revised the article for essential intellectual content and administrative support. All authors read and approved the final manuscript.

\section{Funding}

This work was not funded by any grant.

\section{Availability of data and materials}

All analyzed data are included in this published article and its supplementary information file. The original data are available upon reasonable request to the corresponding author.

\section{Ethics approval and consent to participate}

Not applicable. All data in this study are publicly available.

\section{Consent for publication}

Not applicable.

\section{Competing interests}

The authors declare that they have no competing interests.

\section{Author details}

'Department of Orthopedic Surgery, Zhongnan Hospital of Wuhan University, Wuhan 430071, China. ${ }^{2}$ Department of Pathology, Zhongnan Hospital of Wuhan University, Wuhan 430071, China. ${ }^{3}$ Wuhan University Center for Pathology and Molecular Diagnostics, Wuhan 430071, China.

Received: 13 May 2020 Accepted: 15 September 2020

Published online: 22 September 2020

\section{References}

1. Chang YC, Su CY, Chen MH, Chen WS, Chen CL, Hsiao M. Secretory RAB GTPase 3C modulates IL6-STAT3 pathway to promote colon cancer metastasis and is associated with poor prognosis. Mol Cancer. 2017;16:135.

2. Liu CY, Hsu CC, Huang TT, Lee CH, Chen JL, Yang SH, et al. ER stress-related ATF6 upregulates CIP2A and contributes to poor prognosis of colon cancer. Mol Oncol. 2018;12:1706-17.

3. Zhou R, Zhang J, Zeng D, Sun H, Rong X, Shi M, et al. Immune cell infiltration as a biomarker for the diagnosis and prognosis of stage $\mathrm{I}-\mathrm{II}$ colon cancer. Cancer Immunol Immunother. 2019;68:433-42.

4. Yue B, Qiu S, Zhao S, Liu C, Zhang D, Yu F, et al. LncRNA-ATB mediated Ecadherin repression promotes the progression of colon cancer and predicts poor prognosis. J Gastroenterol Hepatol. 2016:31:595-603.

5. Edwards BK, Ward E, Kohler BA, Eheman C, Zauber AG, Anderson RN, et al. Annual report to the nation on the status of cancer, 1975-2006, featuring colorectal cancer trends and impact of interventions (risk factors, screening, and treatment) to reduce future rates. Cancer. 2010;116:544-73.

6. Center MM, Jemal A, Smith RA, Ward E. Worldwide variations in colorectal cancer. CA Cancer J Clin. 2009:59:366-78.

7. Wang H, Wang X, Xu L, Zhang J, Cao H. A molecular sub-cluster of colon cancer cells with low VDR expression is sensitive to chemotherapy, BRAF inhibitors and PI3K-mTOR inhibitors treatment. Aging (Albany NY). 2019;11: 8587-603.

8. Xu J, Zhao J, Zhang R. Four microRNAs signature for survival prognosis in Colon Cancer using TCGA data. Sci Rep. 2016;6:38306.

9. Yang H, Wu J, Zhang J, Yang Z, Jin W, Li Y, et al. Integrated bioinformatics analysis of key genes involved in progress of colon cancer. Mol Genet Genomic Med. 2019;7:e00588.

10. Jacob H, Stanisavljevic L, Storli KE, Hestetun KE, Dahl O, Myklebust MP. A four-microRNA classifier as a novel prognostic marker for tumor recurrence in stage II colon cancer. Sci Rep. 2018;8:6157.

11. Alonso MH, Ausso S, Lopez-Doriga A, Cordero D, Guino E, Sole X, et al. Comprehensive analysis of copy number aberrations in microsatellite stable colon cancer in view of stromal component. Br J Cancer. 2017;117:421-31.

12. Kanth P, Bronner MP, Boucher KM, Burt RW, Neklason DW, Hagedorn CH, et al. Gene Signature in Sessile Serrated Polyps Identifies Colon Cancer Subtype. Cancer Prev Res (Phila). 2016;9:456-65.

13. Zhang $Q$, Bian $Y$, Zhu Y, Wan L, Kong L, Hu J, et al. Integrative analysis and validation of dysregulated long non-coding RNAs in colon cancer. J Cell Mol Med. 2020;24:2610-21.

14. de Almeida SF, Carmo-Fonseca M. Design principles of interconnections between chromatin and pre-mRNA splicing. Trends Biochem Sci. 2012;37: 248-53.

15. Climente-Gonzalez H, Porta-Pardo E, Godzik A, Eyras E. The functional impact of alternative splicing in Cancer. Cell Rep. 2017;20:2215-26.

16. Xing L, Zhang X, Tong D. Systematic profile analysis of prognostic alternative messenger RNA splicing signatures and splicing factors in head and neck squamous cell carcinoma. DNA Cell Biol. 2019;38:627-38.

17. Wu HY, Peng ZG, He RQ, Luo B, Ma J, Hu XH, et al. Prognostic index of aberrant mRNA splicing profiling acts as a predictive indicator for hepatocellular carcinoma based on TCGA SpliceSeq data. Int I Oncol. 2019; 55:425-38.

18. $X u N$, Ke ZB, Lin XD, Lin F, Chen $S H, W u$ YP, et al. Identification of survivalassociated alternative splicing events and signatures in adrenocortical carcinoma based on TCGA SpliceSeq data. Aging (Albany NY). 2020;12: 4996-5009.

19. Huang $Z G, H e R Q, M o Z N$. Prognostic value and potential function of splicing events in prostate adenocarcinoma. Int J Oncol. 2018;53:2473-87.

20. Xiong $Y$, Deng $Y$, Wang $K$, Zhou H, Zheng $X$, Si L, et al. Profiles of alternative splicing in colorectal cancer and their clinical significance: a study based on large-scale sequencing data. EBioMedicine. 2018;36:183-95. 
21. Lian H, Wang A, Shen Y, Wang Q, Zhou Z, Zhang R, et al. Identification of novel alternative splicing isoform biomarkers and their association with overall survival in colorectal cancer. BMC Gastroenterol. 2020;20:171.

22. Zong Z, Li H, Yi C, Ying $H$, Zhu Z, Wang H. Genome-wide profiling of prognostic alternative splicing signature in colorectal Cancer. Front Oncol. 2018;8:537.

23. Liu J, Li H, Shen S, Sun L, Yuan Y, Xing C. Alternative splicing events implicated in carcinogenesis and prognosis of colorectal cancer. J Cancer. 2018:9:1754-64.

24. Zhang Z, Feng $Q$, Jia C, Zheng P, Lv Y, Mao Y, et al. Analysis of relapseassociated alternative mRNA splicing and construction of a prognostic signature predicting relapse in I-III colon cancer. Genomics. 2020. https:// doi.org/10.1016/j.ygeno.2020.07.002. Online ahead of print

25. Piva F, Giulietti M, Burini AB, Principato G. SpliceAid 2: a database of human splicing factors expression data and RNA target motifs. Hum Mutat. 2012;33: $81-5$.

26. Ryan M, Wong WC, Brown R, Akbani R, Su X, Broom B, et al. TCGASpliceSeq a compendium of alternative mRNA splicing in cancer. Nucleic Acids Res. 2016:44:D1018-22.

27. Yadav S, Bhagat SD, Gupta A, Samaiya A, Srivastava A, Shukla S. Dietaryphytochemical mediated reversion of cancer-specific splicing inhibits Warburg effect in head and neck cancer. BMC Cancer. 2019;19:1031.

28. Sun X, Tian Y, Wang J, Sun Z, Zhu Y. Genome-wide analysis reveals the association between alternative splicing and DNA methylation across human solid tumors. BMC Med Genet. 2020;13:4.

29. Li Z, Chen X, Wei M, Liu G, Tian Y, Zhang X, et al. Systemic analysis of RNA alternative splicing signals related to the prognosis for head and neck squamous cell carcinoma. Front Oncol. 2020;10:87.

30. Devaud C, Tilkin-Mariame AF, Vignolle-Vidoni A, Souleres P, Denadai-Souza A, Rolland C, et al. FAK alternative splice mRNA variants expression pattern in colorectal cancer. Int J Cancer. 2019;145:494-502.

31. Flodrops M, Dujardin G, Busson A, Trouve P, Ka C, Simon B, et al. TIMP1 intron 3 retention is a marker of colon cancer progression controlled by hnRNPA1. Mol Biol Rep. 2020;47:3031-40.

32. Huang $X$, Liu J, Mo X, Liu H, Wei C, Huang L, et al. Systematic profiling of alternative splicing events and splicing factors in left- and right-sided colon cancer. Aging (Albany NY). 2019:11:8270-93.

33. Torrens-Mas M, Hernández-López R, Pons DG, Roca P, Oliver J, Sastre-Serra J. Sirtuin 3 silencing impairs mitochondrial biogenesis and metabolism in colon cancer cells. Am J Phys Cell Phys. 2019;317:C398-c404.

34. Zhang Y, Wang Q, Chen L, Yang HS. Inhibition of p70S6K1 activation by Pdcd4 overcomes the resistance to an IGF-1R/IR inhibitor in Colon carcinoma cells. Mol Cancer Ther. 2015;14:799-809.

35. Lv J, Guo Y, Yan L, Lu Y, Liu D, Niu J. Development and validation of a fiveIncRNA signature with prognostic value in colon cancer. J Cell Biochem. 2019.

36. Chen H, Luo J, Guo J. Development and validation of a five-immune gene prognostic risk model in colon cancer. BMC Cancer. 2020;20:395.

37. Wang $X$, Zhang D, Zhang C, Sun Y. Identification of epigenetic methylationdriven signature and risk loci associated with survival for colon cancer. Ann Transl Med. 2020;8:324.

38. Bechara EG, Sebestyen E, Bernardis I, Eyras E, Valcarcel J. RBM5, 6, and 10 differentially regulate $\mathrm{NUMB}$ alternative splicing to control cancer cell proliferation. Mol Cell. 2013;52:720-33.

39. Zong FY, Fu X, Wei WJ, Luo YG, Heiner M, Cao LJ, et al. The RNA-binding protein QKI suppresses cancer-associated aberrant splicing. PLoS Genet. 2014;10:e1004289.

40. Dvinge H, Kim E, Abdel-Wahab O, Bradley RK. RNA splicing factors as oncoproteins and tumour suppressors. Nat Rev Cancer. 2016:16:413-30.

41. Wan L, Yu W, Shen E, Sun W, Liu Y, Kong J, et al. SRSF6-regulated alternative splicing that promotes tumour progression offers a therapy target for colorectal cancer. Gut. 2019;68:118-29.

42. Chen J, Weiss WA. Alternative splicing in cancer: implications for biology and therapy. Oncogene. 2015;34:1-14.

\section{Publisher's Note}

Springer Nature remains neutral with regard to jurisdictional claims in published maps and institutional affiliations.

\section{Ready to submit your research? Choose BMC and benefit from:}

- fast, convenient online submission

- thorough peer review by experienced researchers in your field

- rapid publication on acceptance

- support for research data, including large and complex data types

- gold Open Access which fosters wider collaboration and increased citations

- maximum visibility for your research: over $100 \mathrm{M}$ website views per year

At BMC, research is always in progress.

Learn more biomedcentral.com/submissions 